\title{
Sino-orbitocranial aspergillosis in a diabetic woman; a case report
}

\author{
Shivanthan MC, Wijesiriwardena B, Ahamed R, Somaratne K, Wickramasinghe C, Silva S \\ National Hospital of Sri Lanka, Colombo, Sri Lanka \\ Correspondence to: Dr.M.C. Shivanthan (mcs627@gmail.com)
}

\section{Introduction}

Sino-orbitocranial aspergillosis manifests with fever, sino-orbital pain and headache that may often elude the diagnosis. We report a case of aspergillosis involving the sinuses with orbital and retrograde intracranial extension involving the skull base causing complete ophthalmoplegia, hemifacial palsy, central retinal artery occlusion and CSF pleocytosis.

\section{Case report}

A 56-year old female with type II diabetes for 6 years presented with sudden loss of vision associated with pain and paralysis of the right eye and right-sided facial weakness, preceded by fever and headache of three weeks. She had worked in Gulf from 1984 1991 and had completed treatment for pulmonary tuberculosis in 2002. She had also suffered a recent left-sided stroke.

Examination revealed a febrile patient with chemosis, complete ophthalmoplegia and ptosis of the right eye with ipsilateral lower motor neuronal facial palsy. Visual acuity in right eye was $0 / 6$ with an insensitive corneoscleral junction with absent pupillary reflexes. The left eye had a cataract with reduced visual acuity. Ophthalmoscopy revealed an ischemic optic fundus in the right side suggestive of a central retinal artery occlusion. Left upper and lower limbs showed upper-motor neurone signs. She had no features of meningism. The examination of other systems was normal.

The initial ESR and CRP were $108 \mathrm{~mm}$ and 8.4 $\mathrm{mg} / \mathrm{dL}$, respectively. WBC was $19.3 \times 10^{9} / \mathrm{L}$ with $93 \%$ neutrophils. Cranial CT showed an old right-sided middle cerebral artery territory infarct and oedema of the soft tissues within the right orbital cavity, maxillary and ethmoidal sinuses.
CSF analysis showed lymphocytes $\left(120 / \mathrm{mm}^{3}\right)$, polymorphs $\left(20 / \mathrm{mm}^{3}\right)$, raised proteins $(53 \mathrm{mg} / \mathrm{dL})$ and normal glucose. No acid-fast bacilli were seen in the CSF. Serum proteins and creatinine were normal and urine had no active sediments. Chest radiograph was also normal. Blood picture was suggestive of an infectious/inflammatory process.

Sino-cranial pathologies namely pyogenic infection, tuberculosis, lymphoma, Wegner's granulomatosis and Tolosa-Hunt syndrome were considered as the possibilities. Broad spectrum intravenous antibiotics, CAT 1 anti-tuberculosis drugs and high dose steroids were empirically commenced. Regional MRI confirmed CT findings with more details. Multi-site biopsies of the sinuses were taken with fiberoptic rhinoscopy. Mantoux, CSF PCR for mycobacteria, C-ANCA, P-ANCA, ANA and HIV antibodies were negative. Bacterial culture of blood and CSF became negative.

Histology of sinus mucosa showed inflammatory changes on $\mathrm{H} \& \mathrm{E}$ staining and fungal staining with methenamine-silver unmasked aspergillosis. Intravenous amphotericin B was commenced and continued beyond two weeks until complete clinical and radiological resolution occurred. Vision and movements in the right eye were, however, not regained. Anti-tuberculosis drugs and steroids were withdrawn and the anti-meningitic antibiotics were continued for the recommended period. The CSF and sinus biopsy specimens sent for fungal culture didn't yield any fungal growth.

Post-treatment imaging confirmed complete radiological resolution of the pathology. She was counseled regarding the permanent nature of the disabilities of her right eye and was referred for early cataract surgery of the left eye. 

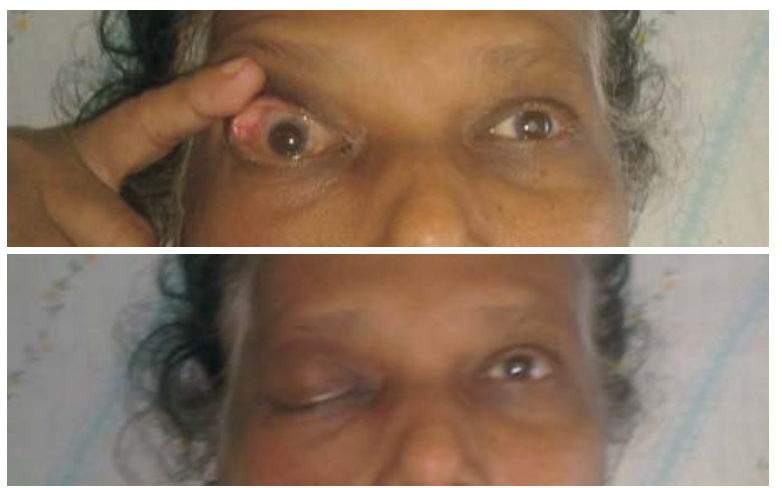

Figure 1: Chemosis and complete ptosis of right eye

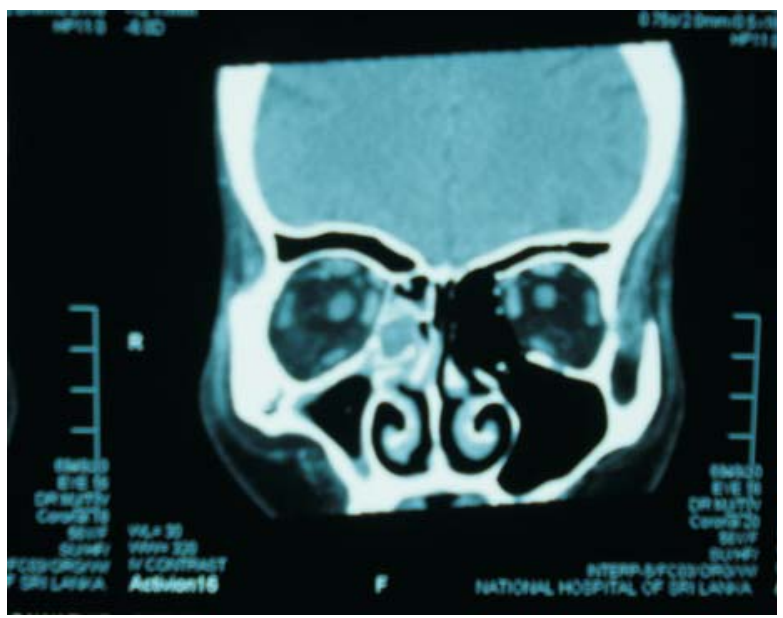

Figure 2: CT scan sagittal view showing rightsided ethmoidal and maxillary sinus soft tissue swelling

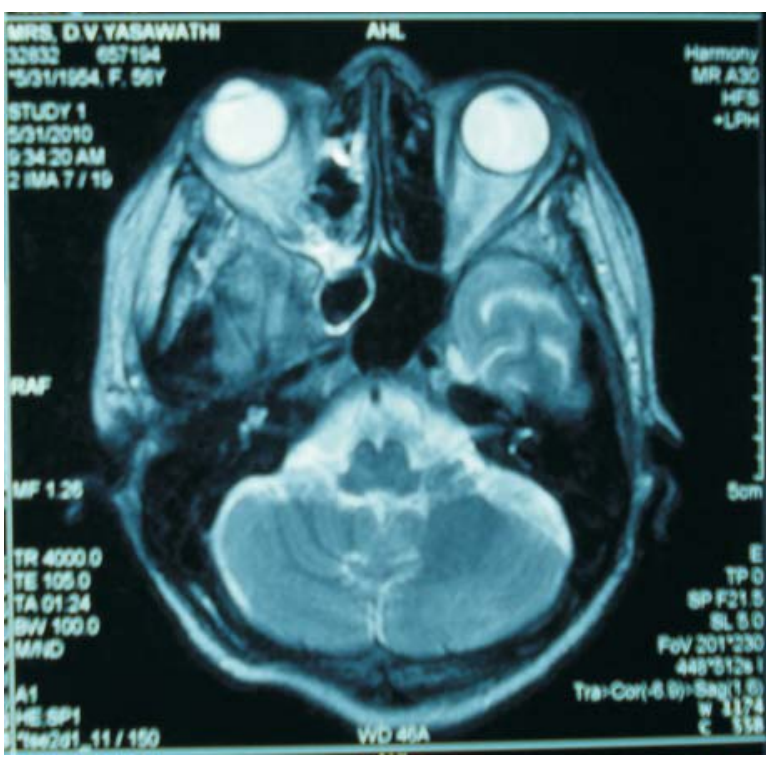

Figure 3: MRI scan coronal view showing soft tissue swelling of orbital cavity contents and proptosis in the right side

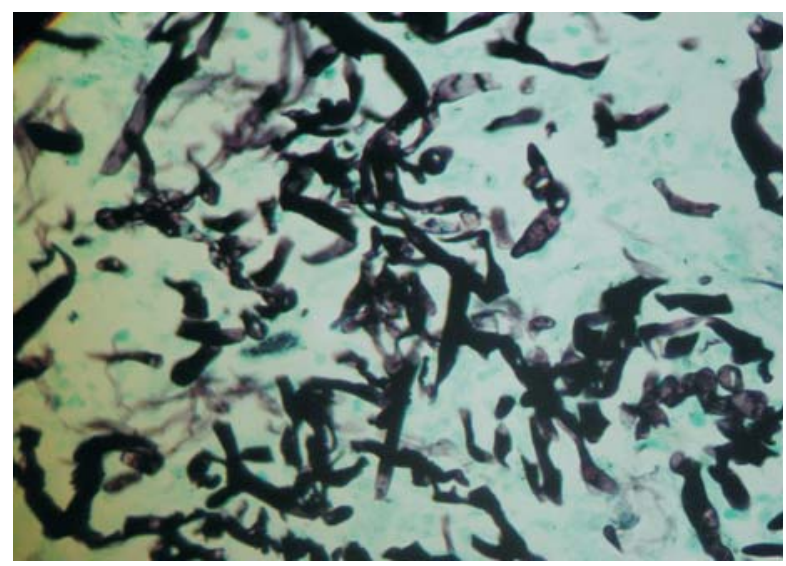

Figure 4: Sinus tissue biopsy stained for fungi showing hyphae with septae and 40 degree branching, diagnostic of Aspergillosis

\section{Discussion}

Detection of infiltrative aspergillosis is usually by microscopic examination of the tissue. Narrow, septate hyphae with acute angle branching are suggestive of aspergillosis. However other fungi, such as Pseudoallescheria boydii, Fusarium spp, Mucorales can cause confusion despite above differentiating features (1). Therefore confirmation is best done by culture as microscopy does not always identify the specific organism though it shows hyphae in tissue. Testing for beta-D glucan and galactomannan which are constituents of aspergillus cell walls, PCR, nucleic acid sequencebased amplification, are still not available in clinical practice. This patient with compatible clinical picture supported by histological evidence of fungal hyphae (2-6) and subsequent clinical response to antifungal treatment (6-9) were sufficient clinical grounds to confirm the diagnosis.

Early diagnosis of the clinical presentations of sinocranial mycoses necessitates timely pattern recognition with laboratory and imaging support.

\section{References}

1. Thomas PA. Current Perspective on Opthalmic Mycosis. Clin Microbiol Rev, 2003; 16: 730-97.

2. Calcott SJ, Livesley N, Nugent RA, Rasmussen SL, Saeed P, Rootman J. Localised invasive Sinorbital Aspergillosis: Characteristic features. British J Ophthalmol 2004; 88: 6817.

Galle Medical Journal, Vol 16: No. 2, September 2011 
3. Sood S, Sharma R, Gupta S, Pathak D, Rishi S. Neuroaspergillosis in an immunocompetent patient. Ind $J$ Med Microbiol, 2007; 25(1): 67-9.

4. Sharada DM,Arunkumar G, Vandana KE,Rao PS. Sinoorbital aspergillosis in a diabetic patient. Ind $\mathrm{J} \mathrm{Med}$ Microbiol, 2006; 24(2): 138-40.

5. Siddiqui AA, Shah AA, Bashir SH. Cranicerebral Aspergillosis of Sinonasal origin in immunocompetent patients. Neurosurgery, 20004; 602-13.

6. Segal BH; Walsh TJ. Current approaches to diagnosis and treatment of invasive aspergillosis. Am J Respir Crit Care Med, 2006Apr 1; 173(7): 707-17.
7. Schwartz S, Ruhnke M, Riband P, Corey L, Driscell T, Cornely OA, et al. Improved outcome in central nervous system aspergillosis; using voriconazole treatment. Blood, 2005; 106: 2641-5.

8. Walsh TJ; Anaissie EJ; Denning DW; Herbrecht R; Kontoyiannis DP; Marr KA; Morrison VA; Segal BH; Steinbach WJ; Stevens DA; van Burik JA; Wingard JR; Patterson TF Treatment of aspergillosis: clinical practice guidelines of the Infectious Diseases Society of America. Clin Infect Dis, 2008 Feb 1; 46(3): 327-60.

9. Boucher HW; Groll AH; Chiou CC; Walsh TJ. Newer systemic antifungal agents: pharmacokinetics, safety and efficacy. Drugs, 2004; 64(18): 1997-2020.

\title{
A case of Dengue fever complicated by acute pancreatitis
}

\author{
Kodisinghe SK, Fernando AHN \\ National Hospital of Sri Lanka, Colombo, Sri Lanka \\ Correspondence to: Dr.Kuleesha Kodisinge (skkodisinghe@gmail.com)
}

\section{Introduction}

Dengue is the most rapidly spreading mosquitoborne viral disease in the world. In the last 50 years, the disease incidence has increased by 30 -fold. It has also become a pandemic, involving the urban and rural populations. Worldwide, an estimated 50 million dengue infections occur annually. The SouthEast Asian region and the Western Pacific region bear $75 \%$ of the current global disease burden. Reported case fatality rates for the South-East Asian region is approximately 1\%(1). Year 2010 had 34097 cases of Dengue in Sri Lanka resulting in 241 deaths. In 2011, 7948 cases of dengue were reported up to mid June, with 69 deaths (2).

We report of a case of Dengue fever complicated by acute pancreatitis. Review of PubMed and Cochrane databases revealed very few reported cases of acute pancreatitis in Dengue (3-6). In South Asia, this is the second reported case of Pancreatitis in Dengue infection (6).

\section{Case report}

A 35 year old male army soldier was transferred to National Hospital of Sri Lanka with fever of 4 days duration with upper abdominal pain. On admission there was flushing, icterus, hepatomegaly with epigastric and right hypochondriac tenderness and bilateral pleural effusions and ascites. There was no bleeding and the patient was haemodynamically stable. On the second day after transfer, his conscious level deteriorated and the GCS was 7/15. There was thrombocytopenia and the dengue IgM antibody was positive. Non contrast CT scan of the brain revealed mild cerebral oedema and the liver functions were of a hepatitis pattern.

He was managed in the Intensive Care Unit with the routine fluid management protocol for dengue, and the anti-liver failure regimen along with N-Acetyl Cysteine. Routine capillary blood sugar measurements detected hyperglycaemia, which was managed with insulin. 